\title{
INTERRELATIONSHIPS AMONG PULMONARY "CAPILLARY" PRESSURE, BLOOD FLOW AND VALVE SIZE IN MITRAL STENOSIS. THE LIMITED REGULATORY EFFECTS OF THE PULMONARY VASCULAR RESISTANCE ${ }^{1}$
}

\author{
By JORGE ARAUJO,2 AND DANIEL S. LUKAS
}

\author{
(From the Cardio-Pulmonary Laboratory of the Department of Medicine, The New York Hos- \\ pital-Cornell Medical Center, New York, New York, and The National Heart \\ Institute, Bethesda, Md.)
}

(Submitted for publication June 18, 1952; accepted September 24, 1952)

Evidence from several sources clearly indicates that the pulmonary vascular bed is abnormal in patients with mitral stenosis. Pathologic studies of the lungs have revealed varying degrees of arteriolar sclerosis, thickening of the basement membrane of the alveolar capillaries, pericapillary edema and fibrosis (1-3). Increased resistance to the flow of blood through the lungs has been confirmed by measurements made with the technique of cardiac catheterization (4-6). In a study of lung biopsy sections obtained at the time of mitral commissurotomy a direct relationship between the magnitude of the pulmonary vascular resistance and the degree of vascular sclerosis was observed (3). In addition to the anatomic alterations, physiologic changes, perhaps in the nature of increased vascular tone, have been implicated. The striking decreases in pulmonary vascular resistance observed within four to six weeks following successful commissurotomy of the mitral valve $(4,6)$ and acutely after the administration of certain vasodilating agents (7) suggest participation of reversible physiologic factors. The pathogenesis of neither the anatomic nor physiologic changes is clearly understood.

Dexter and his co-workers contend that the pulmonary arterioles constrict when the pressure in the capillaries of the lungs attains plasma colloid osmotic levels, as it may in left ventricular failure or in mitral stenosis (8). In studies of mitral stenosis this group of workers have demonstrated an inverse relationship between the size of the calculated mitral valve orifice and the pulmonary

1 This study was aided by grants from the New York Heart Association, and the New York Hospital-Cornell Medical Center Research Fund.

2 W. K. Kellogg Foundation Fellow of The American College of Physicians. arteriolar resistance-the smaller the mitral valve area, the larger the resistance (9). The restriction of pulmonary blood flow by the constricted arterioles has been thought of as protective in nature. Since increases in the rate of flow through a stenotic mitral valve require elevations of left atrial, pulmonary venous, and capillary pressures that frequently exceed plasma protein osmotic pressure it is argued that arteriolar constriction prevents pulmonary edema $(8,9)$.

In this paper certain data obtained by cardiac catheterization of 36 patients with mitral stenosis are presented. Only those observations relative to the interrelationships of mitral valve area and the vascular resistance, "capillary" pressure, blood flow and the occurrence of edema in the lungs are presented. Our data differ from those of Dexter and his co-workers $(8,9)$ in that they failed to demonstrate: 1 ) evidence of pulmonary arteriolar constriction occurring as a response to elevation of the pulmonary "capillary" pressure; 2) any reliable correlation between pulmonary vascular resistance and mitral valve orifice area; 3) that an increased pulmonary vascular resistance prevents increases in pulmonary "capillary" pressure high enough to cause pulmonary edema. More comprehensive data on the pulmonary circulation in mitral stenosis have been published previously (4).

\section{SUBJECTS AND METHODS}

\section{Subjects}

Thirty-six hospitalized patients with mitral stenosis of varying severity were used in this study. The majority were catheterized as part of pre-mitral commissurotomy evaluation and were moderately to severely restricted by their heart disease. All were considered to be compensated at the time of study; those with auricular fibrillation 
and a number of those with normal rhythm were maintained on digitalis.

All but a few subjects had apical systolic murmurs, but clinically and at operation the predominant lesion was mitral stenosis. In 16 (44 per cent) evidence of tricuspid insufficiency was noted in the right atrial pressure tracing obtained at cardiac catheterization. In none was tricuspid stenosis apparent. The high incidence of clinically inapparent tricuspid insufficiency in severe mitral stenosis has been noted previously (4).

Each subject was studied in the resting state, five hours post-absorptive. Sodium amytal $0.2 \mathrm{gm}$. was administered one-half hour prior to the procedure.

\section{Methods}

The technique of cardiac catheterization and the analytic methods were reported previously (4). Pulmonary "capillary," pulmonary arterial, right ventricular pressures and cardiac output were determined at rest. These observations were repeated during the last two minutes of a five-minute exercise, which consisted of flexion and extension of the legs in a bicycling manner at the rate of one cycle per two seconds.

The area of the mitral valve orifice (MVA) was calculated by the method of Gorlin and Gorlin (10) except that the diastolic filling period, during which blood flows across the mitral valve, was determined from the right ventricular or pulmonary arterial pressure tracing. In 21 patients where sufficient data were available the MVA was calculated during both rest and exercise (Table I). The average difference between the values from the two calculations was $0.12 \mathrm{sq}$. $\mathrm{cm}$; the range of difference was 0.01 to $0.34 \mathrm{sq}$. $\mathrm{cm}$. The close agreement between the two sets of calculated valve areas affords an internal check on the reliability of the methods of obtaining the data and the calculations. This, however, does not vali-

\section{TABLE I}

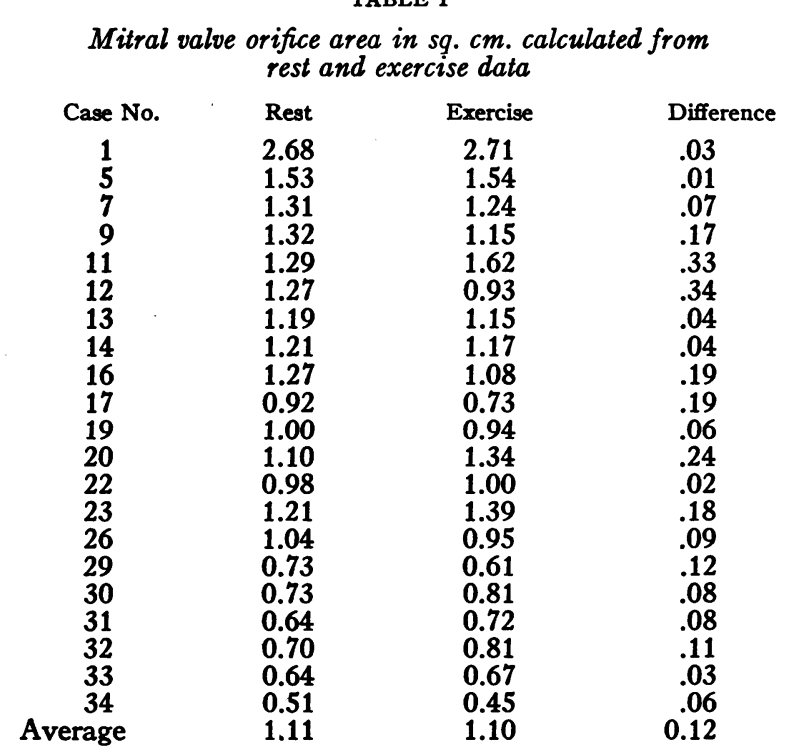

date the Gorlin formula since it may yield considerably inaccurate values in the presence of marked mitral insufficiency. Experience with this and other groups of patients with small degrees of mitral insufficiency has indicated that the MVA usually agrees closely with the area determined at autopsy or commissurotomy. The Gorlin formula has been used here since it is the only available method of estimating MVA in the living patient and because it has been used extensively by other workers. The calculated MVA was related to body surface area (BSA) and expressed as MVA index. The pulmonary capillary pressure is regarded by many $(10-13)$ as a reasonable estimate of the pressure in the pulmonary veins and left atrium and is used interchangeably with the latter in the discussion. The true pressure in the capillaries of the lungs is thought to be a few $\mathrm{mm}$. $\mathrm{Hg}$ higher than the "PC" pressure (12).

The pulmonary vascular resistance (PVR) was determined by the formula of Apéria (14):

where

$$
\mathrm{PVR}=\frac{(\mathrm{PAm}-\text { "PC"m }) \times 1.332 \times 60}{\mathrm{CO}}
$$

PVR = pulmonary vascular resistance in dynes-sec.cm. ${ }^{-5}$

PAm $=$ mean pulmonary arterial pressure in $\mathrm{mm} . \mathrm{Hg}$ "PC" $\mathrm{m}=$ mean pulmonary "capillary" pressure in $\mathrm{mm}$. $\mathrm{Hg}$

$\mathrm{CO}=$ cardiac output in $\mathrm{L} . / \mathrm{min}$.

This measurement includes the resistance of the pulmonary capillaries since the pressure decrement from pulmonary artery to vein is used.

\section{RESULTS}

\section{" $P C$ " pressure at rest and exercise}

In two subjects, resting "PC" pressure was almost normal; the MVA index was greater than $1.4 \mathrm{sq}$. cm. in both. In the remainder of the subjects MVA indices were below $1.4 \mathrm{sq} . \mathrm{cm}$. and there was an increase in the resting "PC" pressure to a strikingly similar level (Table II). The mean value was $28 \mathrm{~mm}$. $\mathrm{Hg}$ with a narrow standard deviation of $\pm 3.2 \mathrm{~mm}$. Despite variations in the PVR the hydrostatic pressure in the pulmonary capillaries remained in the range of plasma colloid osmotic pressure (i.e., 25 to $30 \mathrm{~mm}$. $\mathrm{Hg}$ ) and significant pulmonary edema was not present in any of the subjects.

The adjustment in "PC" pressure was adequate for the maintenance of a normal cardiac index at rest when the MVA index was greater than 0.9 sq. $\mathrm{cm}$. As the mitral valve narrowed below this figure the cardiac index declined directly $(r=$ $0.897 \pm 0.037$ ) (Figure 1). To facilitate normal 
TABLE II

Physical characteristics, mitral valve area indices, cardiac indices, pulmonary "capillary" pressures and vascular resistances during rest and exercise in mitral stenosis

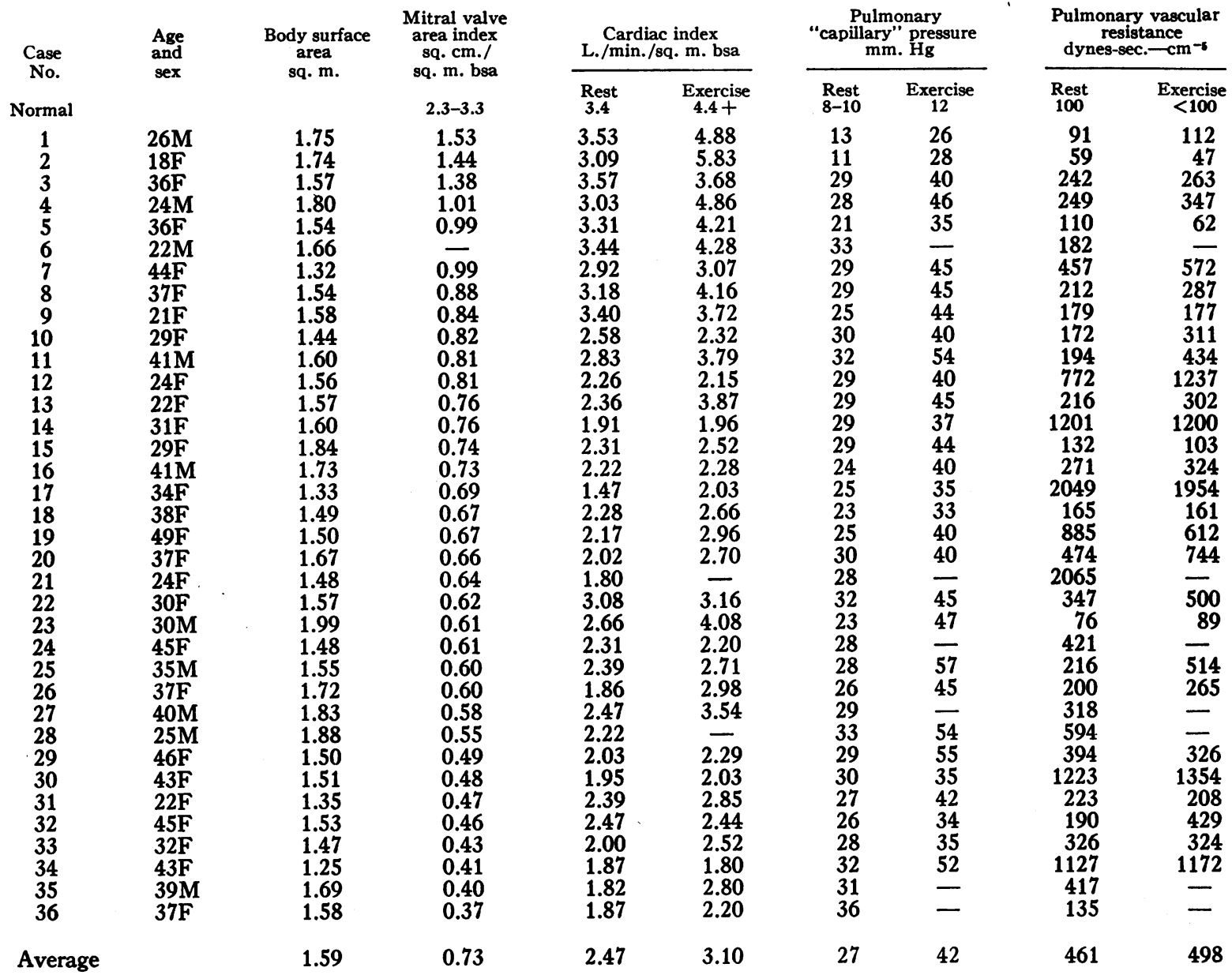

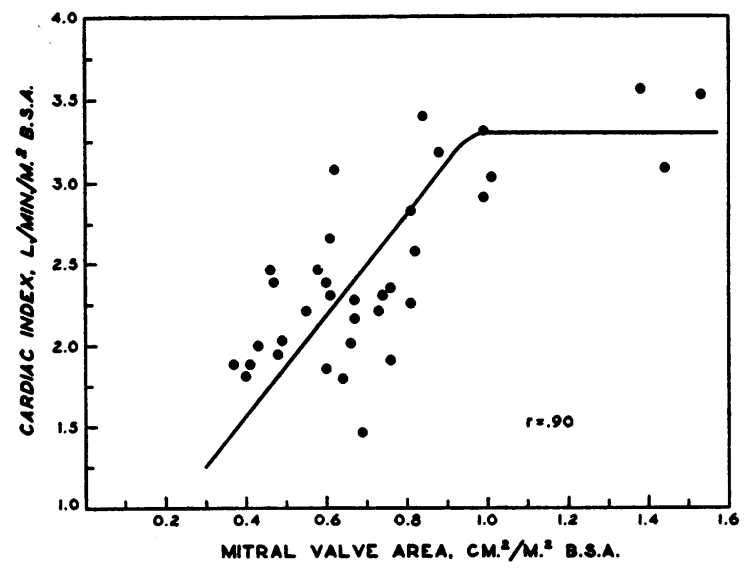

Fig. 1. Relationship of Cardiac Index to Mitral Valve Area Index in Mitral Stenosis blood flow across such small mitral orifices, "PC" pressures larger than those encountered would have been required.

During exercise "PC" pressure rose abruptly to levels well above plasma protein osmotic pressure. The degree of increase above the resting value did not correlate with the PVR ( $r=$ $-0.310 \pm 0.168$ ). Many subjects with greatly increased PVR had marked elevation of "PC" pressure with exercise. It was apparent that increase in cardiac output was not solely responsible for this change since only 15 subjects had adequate increases in output of one L./min./sq. M. BSA or more. In the remainder, cardiac index increased slightly, not at all, or even decreased. Marked tachycardia was frequent during exercise. Since 
the diastolic period of the cardiac cycle is shortened with tachycardia, "PC" pressure also rose in association with restriction of the time allotted in the cardiac cycle for the flow of blood across the stenotic mitral valve (15).

Although "PC" pressure was well elevated above plasma colloid osmotic pressure during the five minute exercise period, acute pulmonary edema occurred in only one subject (subject 16). This patient developed marked orthopnea, cough and many moist rales in his lungs during the fourth minute of exercise. His "PC" pressure was 54 mm. Hg and PVR 594 dynes-sec.-cm. ${ }^{-5}$, a value six times normal. The occasional appearance of a few rales at the lung bases was the only sign of pulmonary edema in the other subjects.

\section{Pulmonary vascular resistance ( $P V R$ )}

The PVR varied from normal to 20 times normal (Table II). Correlation between PVR and mitral valve size was poor in both a direct and logarithmic comparison $(r=-0.316 \pm 0.154$ and $r=-0.435 \pm 0.139$, respectively). A large PVR, however, was most frequently but not consistently encountered among patients with very small mitral valve orifices. As example: PVR among subjects with MVA indices of 0.4 to 0.5 varied from normal to 1,223 dynes-sec.-cm. ${ }^{-5}$.

There was an inverse logarithmic correlation between the cardiac output and PVR during both rest $(r=-0.707 \pm 0.085)$ and exercise $(r=$
$-0.757 \pm 0.082$ ) (Figure 2). However, the degree of change in cardiac output with exercise from resting levels did not correlate well with the PVR $(r=-0.487 \pm 0.133)$. In general an increase of PVR was associated with depression of blood flow below that required by the metabolic needs of the body but not necessarily with inability to increase cardiac output with exercise.

\section{DISCUSSION}

Depending upon the size of the valve orifice mitral stenosis presents varying degrees of obstruction to the flow of blood from left atrium to left ventricle. The degree of obstruction depends also on the usual blood flow requirements of the individual and therefore on his size. It is apparent that a valve orifice of $1 \mathrm{sq}$. $\mathrm{cm}$. is physiologically more tolerable in a small child with a body surface area of 1 sq. M. than it would be in a large well-developed male with a 2 sq. M. body

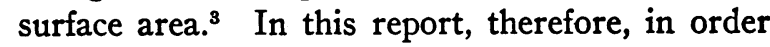

${ }^{3} \mathrm{~A}$ child with a 1 sq. $M$. body surface area has a resting cardiac output in the range of $3.4 \mathrm{~L} . / \mathrm{min}$.; a man with a 2 sq. M. body surface area has an output of 6.8 L./min. The effect of a mitral stenosis with an orifice area of $1.0 \mathrm{sq}$. $\mathrm{cm}$. on the "PC" pressure of each can be calculated from the Gorlin formula :

$$
\text { MVA }=\frac{\frac{C O}{\mathrm{DFP}}}{31 \sqrt{\text { "PC" }-5}}
$$

If a diastolic filling period of $30 \mathrm{sec}$. is assumed the " $\mathrm{PC}$ "
REST

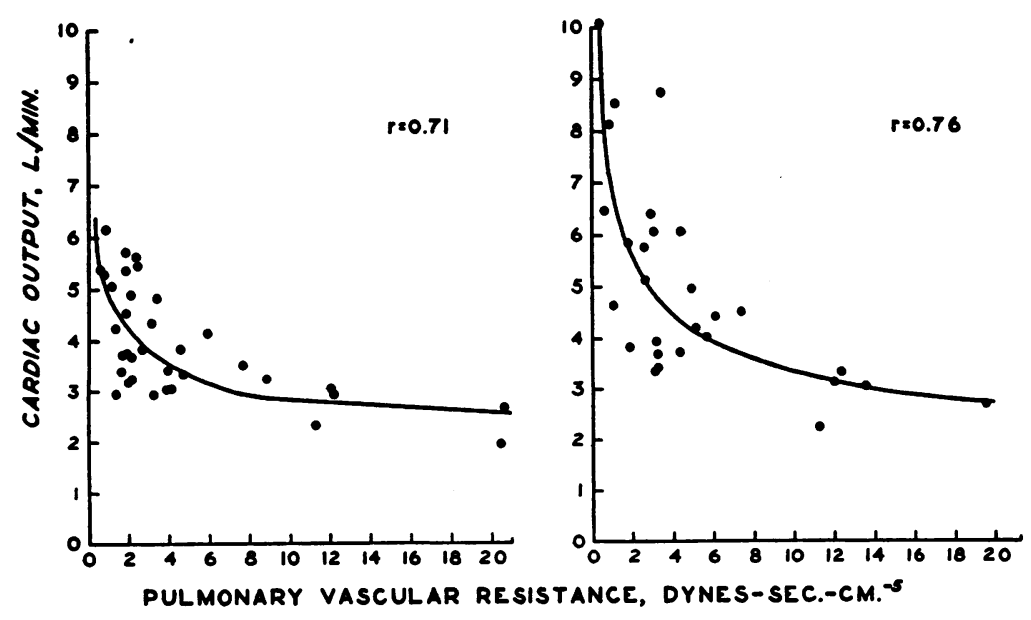

Fig. 2. Relationship of Cardiac OUtput to Pulmonary Vascular Resistance During Rest and Exercise in Mitral Stenosis 
to compare the effects of stenosis among subjects of varying size the MVA was referred to the body surface area and expressed as MVA index in the same way as is conventional for the cardiac output.

With MVA indices greater than $1.4 \mathrm{sq} . \mathrm{cm}$. only modest increases of left atrial, pulmonary venous, capillary and arterial pressures are required to maintain a normal cardiac index at rest. With smaller mitral orifices the maintenance of cardiac output is accomplished at the expense of elevation of pulmonary venous pressure to plasma colloid osmotic levels (i.e. 25 to $30 \mathrm{~mm}$. $\mathrm{Hg}$ ). Since the resting pulmonary venous pressure remains selectively fixed at $28 \pm 3.2 \mathrm{~mm}$. $\mathrm{Hg}$, progressive narrowing of the MVA index below 0.9 sq. $\mathrm{cm}$. is associated with progressive and marked diminution in cardiac output.

It appears therefore that in severe mitral stenosis selective fixation of the resting pulmonary venous pressure in the plasma protein osmotic range is effected by reduction of cardiac output. The mechanism by which cardiac output is reduced is not known. Dexter (8), Lewis (9) and their associates believe that the increased flow resistance of the pulmonary arterioles is responsible. In the present study an inverse correlation between cardiac output and PVR was observed. It is not likely, however, that lowering of blood flow is accomplished by an increased PVR since output was similarly depressed among patients with identical MVA regardless of the degree of PVR change. Nor can regulation of cardiac output be ascribed to inability of the right ventricle to pump blood since many of these patients were able to increase their output with exercise. It is possible that the cardiac output is regulated by reflexes originating from pressure receptors in the left atrium or the pulmonary veins.

Other investigators $(5,6)$ have not observed as close an agreement in resting " $\mathrm{PC}$ " pressure among subjects with mitral stenosis as reported here. It is possible that the state of cardiac compensation was more variable in their patients. This is true in the case of one report in which several patients with failure were included (6). None of

pressure in the child at rest would be $18 \mathrm{~mm}$. $\mathrm{Hg}$ and in the man $58 \mathrm{~mm}$. Hg. The effect of a stenotic mitral orifice of similar size on the pulmonary circuit pressures in these different-sized individuals is obviously marked. the present group of subjects was decompensated at the time of cardiac catheterization.

It is of interest that the direct measurements of left atrial pressure in 10 patients with severe mitral stenosis reported by Bland (16) averaged $32 \mathrm{~mm}$. Hg (range : 27 to $39 \mathrm{~mm}$.), a value only slightly higher than the average "PC" pressure in our patients. These measurements, however, were made in the open chest at time of operation and are not accompanied by a zero reference level.

Experimentally edema of the lungs occurs when the pulmonary venous pressure attains plasma colloid osmotic levels $(17,18)$. In the present study the absence of significant pulmonary edema with pulmonary venous pressures in such a range at rest, and well above during brief exercise, is a matter of considerable interest. These facts suggest that the alveolar-capillary structures in mitral stenosis are not normally permeable to fluid. The anatomic alterations (i.e. capillary basement membrane thickening and pericapillary fibrosis) that very commonly exist in these structures probably impede transudation of fluid from the pulmonary capillaries out into the alveoli. This is suggested by the frequency with which interstitial pulmonary edema without edema fluid in the alveolar spaces is observed in lung biopsy sections from patients with mitral stenosis (3).

The data of Gorlin and his co-workers (5) provide additional evidence of decreased permeability of the alveolar membranes to fluid. In their patients with mitral stenosis pulmonary edema at rest was noted only in those with pulmonary "capillary" pressures averaging $40 \mathrm{~mm}$. Hg (a value considerably in excess of plasma protein osmotic pressure): whereas those with pulmonary "capillary" pressures in the range of osmotic pressure (average $24 \mathrm{~mm} . \mathrm{Hg}$ ) did not manifest pulmonary edema at rest. From these observations it appears that regulation of the resting pulmonary venous pressure at the plasma protein osmotic level and decreased alveolar membrane permeability to fluid combine to prevent the development of pulmonary edema in the patient with severe mitral stenosis. Clinically detectable pulmonary edema is produced only by prolonged elevations of pulmonary venous pressure well in excess of plasma protein osmotic pressure.

This study sheds no light on the pathogenesis of the increased resistance to flow of the pulmonary 
vessels. It is evident that very small mitral orifices predispose to the development of pulmonary vascular changes but are not always associated with them. In view of the frequently normal and wide range of values for PVR in association with "PC" pressures of 25 to $30 \mathrm{~mm}$. $\mathrm{Hg}$, there is little evidence to indicate that arteriolar contriction occurs as a response to elevations of pulmonary venous pressure to the plasma protein osmotic range, as has been suggested previously $(8,9)$. The close relationship between measured PVR and degree of vascular sclerosis observed in lung biopsy sections (3), suggests that these structural changes in the vessels are primarily responsible for the resistance to flow.

Although there is some tendency of a large resistance to damp increases of pulmonary blood flow during exercise, large changes in pulmonary venous pressure are not prevented. Indeed the degree of increase of both blood flow and pulmonary venous pressure with exercise cannot be related reliably to the state of the PVR. In the light of these observations the concept of an increased PVR performing an active protective function in mitral stenosis must be held in doubt.

It has been pointed out repeatedly that pulmonary vascular changes cause a disproportionate rise of arterial pressure with respect to the venous pressure in the lungs (4-6). Right ventricular work is consequently increased. In some instances more work is expended by the right ventricle in driving blood through the pulmonary vessels than through the stenotic mitral valve (4). Large increments in PVR thereby accelerate the process of right ventricular hypertrophy, dilatation and failure.

\section{SUM MARY}

The maintenance of blood flow across the stenotic mitral valve is accompanied by an increase in the left atrial and pulmonary venous pressure. In a group of patients with severe mitral stenosis but not in cardiac failure pulmonary "capillary" pressure at rest was found to be fixed within the range of the plasma protein osmotic pressure. Fixation of the pulmonary "capillary" pressure was accomplished at the expense of the resting cardiac output which varied directly with the size of the mitral orifice. The mechanism by which reduction of cardiac output (needed for regulation of pulmonary “capillary" pressure) was accomplished is not known. It was not achieved by an increased pulmonary vascular resistance since there was no correlation between mitral valve size and pulmonary vascular change. The latter varied widely among individuals with similar valve areas.

Large increases in pulmonary "capillary" pressure occurred during exercise and were not prevented by an increased pulmonary vascular resistance. There was no evidence that pulmonary arteriolar constriction occurs when the pulmonary "capillary" pressure attains plasma protein osmotic levels. Despite pulmonary "capillary" pressures at and above such levels detectable alveolar edema was rare. This suggests that changes in the alveolar membrane rather than in the pulmonary arterial tree protect against pulmonary edema. The increased pulmonary vascular resistance of mitral stenosis is primarily the result of anatomic changes in the vessels.

\section{REFERENCES}

1. Parker, F., Jr., and Weiss, S., The nature and significance of the structural changes in the lungs in mitral stenosis. Am. J. Path., 1936, 12, 573.

2. Larrabee, W. F., Parker, R. L., and Edwards, J. E., Pathology of intrapulmonary arteries and arterioles in mitral stenosis. Proc. Staff Meet. Mayo Clin., 1949, 24, 316.

3. Lukas, D. S., Pearce, J. M., and Glenn, F. S., The relation of anatomic to physiologic changes in the pulmonary vessels in mitral stenosis. In preparation.

4. Lukas, D. S., and Dotter, C. T., Modifications of the pulmonary circulation in mitral stenosis. Am. J. Med., 1952, 12, 639.

5. Gorlin, R., Haynes, F. W., Goodale, W. T., Sawyer, C. G., Dow, J. W., and Dexter, L., Studies of the circulatory dynamics in mitral stenosis. II. Altered dynamics at rest. Am. Heart J., 1951, 41, 30.

6. Draper, A., Heimbecker, R., Daley, R., Carroll, D., Mudd, G., Wells, R., Falholt W. Andrus, E. C., and Bing, R. J., Physiologic studies in mitral valvular disease. Circulation, 1951, 3, 531.

7. Lukas, D. S., and Araujo, J., personal observations.

8. Dexter, L., Dow, J. W., Haynes, F. W., Whittenberger, J. L., Ferris, B. G., Goodale, W. T., and Hellems H. K., Studies of the pulmonary circulation in man at rest. Normal variations and the interrelations between increased pulmonary blood flow, elevated pulmonary arterial pressure, and high pulmonary "capillary" pressures. J. Clin. Invest., 1950, 29, 602.

9. Lewis, B. M., Gorlin, R., Houssay, H. E. J., Haynes, F. W., and Dexter, L., Clinical and physiological 
correlations in patients with mitral stenosis. V. Am. Heart J., 1952, 43, 2.

10. Gorlin, R., and Gorlin, S. G., Hydraulic formula for calculation of the area of the stenotic mitral valve, other cardiac valves, and central circulatory shunts. I. Am. Heart J., 1951, 41, 1.

11. Gorlin, R., Dow, J. W., Hellems, H. K., and Dexter, L., Pulmonary "capillary" pressure as an index of left atrial mean pressure in dogs. Am. J. Med., 1950, 8, 396.

12. Hellems, H. K., Haynes, F. W., Dexter, L., and Kinney, T. D., Pulmonary capillary pressure in animals estimated by venous and arterial catheterization. Am. J. Physiol., 1948, 155, 98.

13. Lagerlöf, H., and Werkö, L., Studies on the circulation of blood in man. VI. The pulmonary capillary venous pressure pulse in man. Scandinav. J. Clin. \& Lab. Invest., 1949, 1, 147.
14. Apéria, A., Hemodynamical studies. Skandinav. Archiv. f. Physiol., 1940, 83, Supplement 16.

15. Gorlin, R., Lewis, B. M., Haynes, F. W., Spiegl, R. J., and Dexter, L., Factors regulating pulmonary "capillary" pressure in mitral stenosis. IV. Am. Heart J., 1951, 41, 834.

16. Bland, E. F., Surgery for mitral stenosis. A review of progress. Circulation 1952, 5, 290.

17. Campbell, G. S., Haddy, F. J., Adams, W. L., and Visscher, M. B., Circulatory changes and pulmonary lesions in dogs following increased intracranial pressure and the effect of atropine upon such changes. Am. J. Physiol., 1949, 158, 96.

18. Haddy, F. J., Campbell, G. S., and Visscher, M. B., Pulmonary vascular pressures in relation to edema production by airway resistance and plethora in dogs. Am. J. Physiol., 1950, 161, 336. 